\title{
Automation of Quantity Take-off for Modular Construction
}

\author{
Hongru ZHAO ${ }^{1}$, Hexu LIU $^{1} *$ and Mohamed AL-HUSSEIN ${ }^{1}$ \\ ${ }^{1}$ Department of Civil and Environmental Engineering, University of Alberta, Canada \\ *Corresponding author's e-mail: hexu@ualberta.ca
}

\begin{abstract}
Quantity takeoff, serving as a foundation for the downstream tasks in the construction management, is a repetitive work. However, this process in current practice involves massive manual interventions, which is extremely time consuming and highly error-prone. This is partially due to the fact that incorporating cost breakdown structure formulated according to industry companies' classification system into BIM still remains a challenge. This study thus exploits a methodology which allows construction practitioners to obtain quantity takeoff in an automatic manner. The main concept is to pre-load the unique classification information into the BIM model such that the quantity of materials in a given BIM model can be extracted and stored into a database (Excel Sheet) automatically in according with the preloaded classification system. Besides this, the unique classification information, along with formulas for derivedquantities, is front-loaded into the Excel Sheet database. As a result, the explicitly extracted quantities are converted by the preloaded formulas to the required format for the purpose of ordering and purchasing. A prototype system is developed based on Autodesk Revit through Revit Application Programming Interface. A case study of a modularized house reveals that a considerable amount of time saving and accuracy increasing of project estimation are achieved as a result of achieving the quantity takeoff automation.
\end{abstract}

\section{KEYWORDS}

Modular construction; Quantity Take-off; Building Information Modelling

\section{INTRODUCTION}

Modular construction refers to a substantial portion of building units are fabricated or assembled in an off-site manufactory and then transported to the construction field for site erection (Pasquire, 2002). Modular construction had experienced a strong growth with an average of 12\% from 1991 to 2001(Becker, 2003). For such promising and high-tech industry, most companies still adhere to traditional means of quantity takeoff, which is performed by using engineering rules, paper-and-pencil methods, and calculators. Accordingly, the work of quantity takeoff on a typical $72 \mathrm{ft} \times 12 \mathrm{ft}$ module project takes more than two hours by taking this manual approach. Besides that, the manual quantity takeoff is a highly error-prone and cumbersome task, and a quantity takeoff revision is also a nightmare for estimators due to plan changes. The current highly developed technology, which is Building Information Modelling (BIM), provides a more intelligent approach to perform the quantity takeoff than manual work. Generally, BIM is the process of modelling a three-dimensional (3D) data set and the intelligent and computable data stored in BIM model can be shared and utilized by various stakeholders. BIM also facilitates the 
estimation, procurement, and construction activities by creating accurate virtual model with precise geometry and data (Eastman et al, 2008). In terms of quantity takeoff, a large amount of commercial and in-house software is developed on basis of BIM platform. Among all of the software, "Autodesk Quantity Takeoff", which is developed by Autodesk, is the most wellknown quantity takeoff software in the current industry. It enables the cost estimators to read and extract information (geometry, images and data) from building information modelling tools, such as Revit Architecture, Revit Structure and Revit MEP. After importing the data from BIM authoring software, Autodesk Quantity Takeoff is able to automatically measure, count and price building objects in minutes, and the takeoff results can be exported into different formats, such as Microsoft Excel and DWF file format (Autodesk Quantity Takeoff, 2015). However, the high complexity, tedious initialization setting, and limited customization restrain the utilization in modular construction. Consequently, this research develops an add-on through the BIM platform (Revit) to perform the quantity takeoff in an automatic manner. The information and building component quantities are extracted from the BIM model and imported into a database (Excel Spreadsheet) directly, and what an estimator needs to do is only activating the add-on and selecting an Excel template. By implementing the add-on, the whole process can be done in less than two minutes, which is a dramatic saving, compared to the current practice, and in the meanwhile, the accuracy is increased to $100 \%$.

\section{METHODOLOGY}

The overview of the methodology is shown in Figure 1. The main process is to front-load unique classification system regarding material information (e.g. part number and unit number) into BIM 3D model and database template, and extract the material quantity in accordance with the part number and unit number to the database. A certain portion of directly extracted quantities are inadequate to be utilized for inventory, and the front loaded formulas in the database are featured to process the quantities. The detailed explanation is given in the following sections.

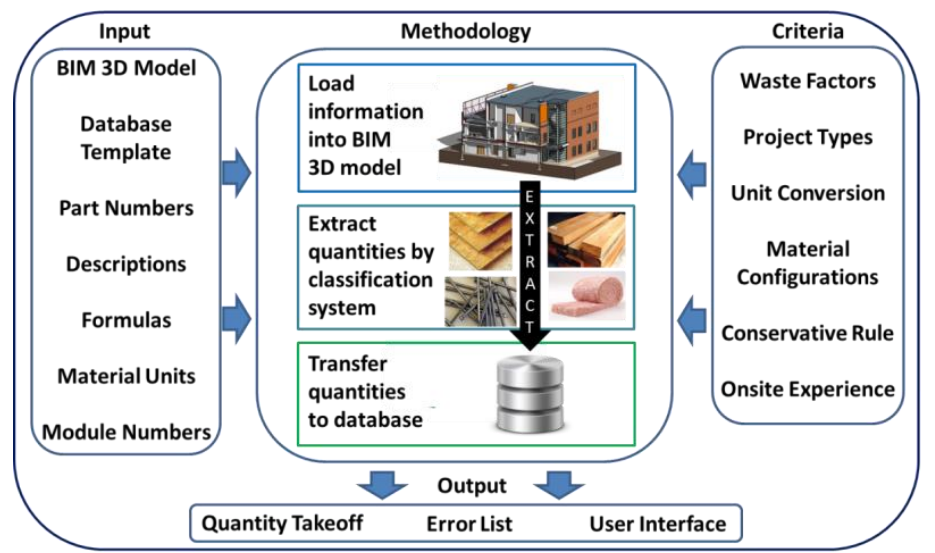

Figure 1. Overview of methodology of quantity takeoff

\section{Preload Revit Model with unique classification system}

The concept of automatic quantity takeoff is to integrate BIM software and database coherently through the unique classification system and quantify the items in the BIM model into the database template. The "bridge" used to link two ends is part number, which is an identifier of an item. To extract the quantities of materials, part numbers are preloaded into BIM 3D model and assigned to each item. However, under certain circumstances, part number is not sufficient to 
classify items for modular construction. The characteristic of this construction method is that a single project comprises multiple independent modules. To distinguish the modules, each one is assigned a property referred to as "Unit Number", and all the materials belonging to the same module have the identical "Unit Number".

\section{Quantification Measurement}

To facilitate the quantity extraction, material usage is quantified by using various measurements, such as each, linear length and contact area. In this study, materials are separated into three categories (Figure 2) with regard to different measurement units and information front-loaded.

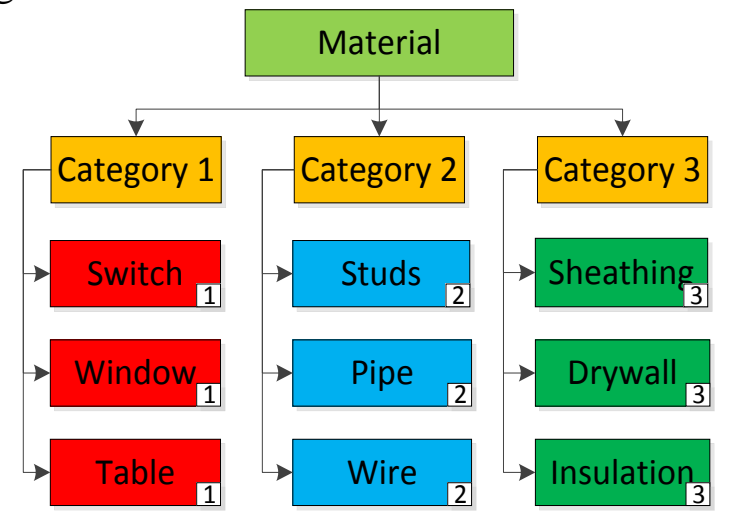

Figure 2. Material category classification

- Category 1 (C1): Materials counted by each (For example, doors and windows).

- Category 2 (C2): Materials counted by linear length (For example, lumber and pipe).

- Category 3 (C3): Materials counted by area (For example, OSB sheathing and drywall).

\section{Format Excel Template}

A standardized database template is prerequisite to automate the quantity takeoff. Generally, the database template contains all the cost items that are needed for module construction; it thus could be commonly modified from a company's "Inventory File". At least five properties are belonging to the database, "Part Number", "Description", "Unit", "Module Number", and "Quantity". "Part Number" and "Module Number" are the primary and secondary indicators to conduct the quantity extraction. "Description" and "Unit" are supplementary properties for comprehension of a certain material. All the properties except "Quantity" are constant and front loaded into database, and the "Quantity" is kept blank to hold the quantity extracted from the BIM 3D model. In most cases, the content in the database template needs to be duplicated for a few times, and the number of duplication should be equal to the quantity of the units (modules) in a model. After each copy of the content, different "Module Numbers" is assigned.

However, this database structure is still not fully feasible for quantity takeoff due to three considerations, inevitable waste, real construction method, and unit conversion. Among all three material categories $(\mathrm{C} 1)$, the first category items do not need any further processes after being extracted, and the quantities are regarded as $100 \%$ accurate. For example, "five windows and four doors" are extracted from the model, and that precisely indicates the project requires five windows and four doors. However, the quantities of second category (C2) have less accuracy than the first category, since the $\mathrm{C} 2$ materials always need extra processing works in reality, such 
as lumber and pipe cutting. The third category (C3) has the lowest accuracy, since C3 materials usually need two-dimensional cut to have suitable sizes. The extra processes of category 2 and category 3 materials cause inevitable waste, which leads to a significant deviation on quantity estimation. Besides that, the system configuration of BIM software is unable to quantify the area of Category 3 (C3) materials properly referring to the real construction demand. A wall surface of Autodesk Revit, which is typical BIM software, is selected for explanation. The wall comprises multiple layers of Category 3 materials, such as insulation and house wrap (Figure 3(b)), and the default assumption of Revit is that Category 3 (C3) materials hosted by a wall have the equivalent contact area as the wall surface area (Figure 3(a) Equation 1). However, while installing home wrap onto a window opening, the part overlaid on top of the opening is broken by a crossing cut, and four formed triangles are pushed inside of the opening for sealing window edges, and the surplus parts are commonly wasted (Figure 4 (a)). Hence the extracted quantity $\left(A_{h w}\right)$ of home wrap should be less than the actual demand $\left(A_{H W}\right)$ if windows exist on a wall. Conversely, the insulation strip is only filled in the spaces between studs rather than on top of the studs (Figure $4(\mathrm{~b})$ ), so the extracted area $\left(A_{i}\right)$ must be larger than the real need $\left(A_{I}\right)$.

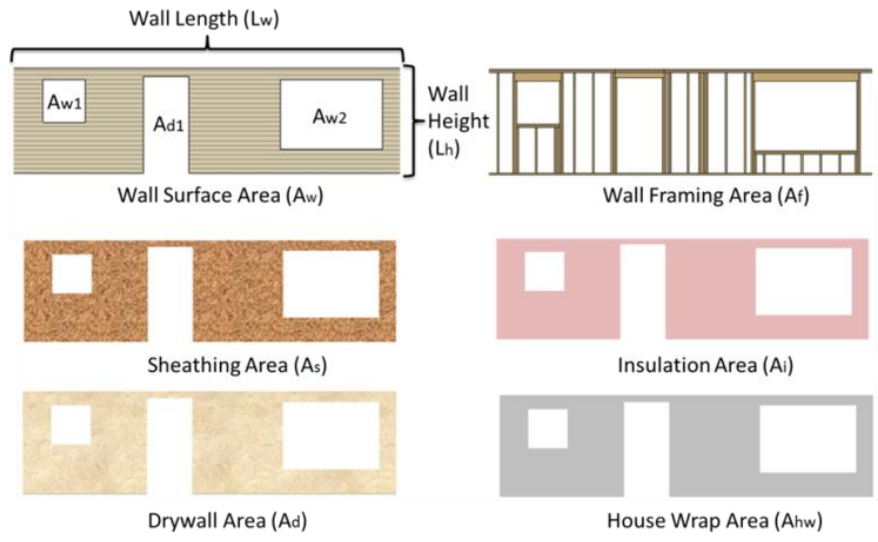

(a)

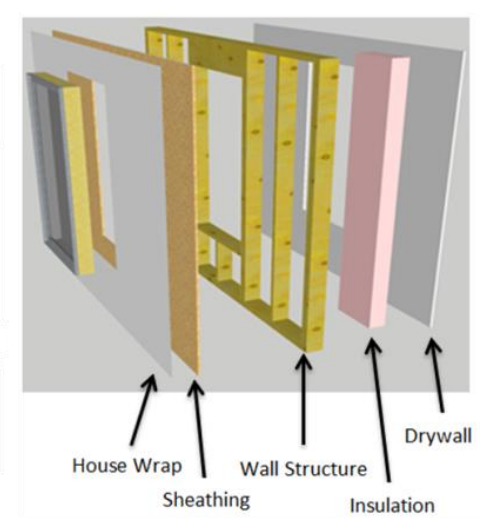

(b)

Figure 3. Wall material areas are the same as the wall surface area

$$
\begin{gathered}
A_{w s}=A_{s}=A_{i}=A_{d}=A_{h w}=L_{w} \times H_{w}-A_{w 1}-A_{w 2}-A_{d 1} \\
A_{h w}=L_{w} \times H_{w}-A_{w 1}-A_{w 2}-A_{d 1} \\
A_{H W}=L_{w} \times H_{w}>A_{h w} \\
A_{i}=L_{w} \times H_{w}-A_{w 1}-A_{w 2}-A_{d 1} \\
A_{I}=A_{i}-A_{f}
\end{gathered}
$$

Where $A_{w s}$ is the wall surface area; $A_{s}$ is the extracted sheathing area; $A_{i}$ extracted insulation area; $A_{I}$ represents demanded insulation area; $A_{d}$ denotes extracted drywall area; $A_{h w}$ represents the extracted house wrap Area; $A_{H W}$ is the demanded house wrap area; $L_{w}$ and $H_{w}$ are the length and height of wall, respectively; $A_{w 1}$ is the area of first window opening; $A_{w 2}$ denotes area of second window opening; and $A_{d 1}$ represents area of first door opening.

In view of the aforementioned scenarios, it is advisable to be cautious of the variability of material usage, and issue more or less on top of the extracted quantities appropriately. In practical, project estimators often assign extra percentages to material quantities for conservative, and most of the percentages are decided by on-site observation and experience. 


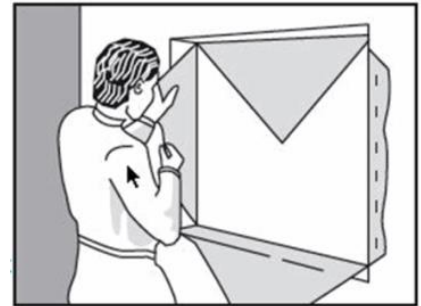

(a)

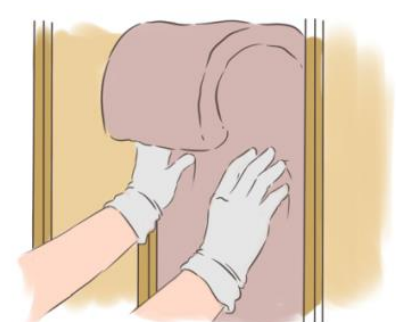

(b)

Figure 4. House wrap membrane and insulation installation ("Tyvek Housewrap Breather Membrane for wall condensation protection" 2014) ("Basement Wall Insulation Blanket Viewing Gallery" 2014)

Even taking no account of the previously considerations, a certain portion of quantities extracted directly from a model are still not qualified for cost estimation or purchasing purpose, due to a fact of that the default units of materials in BIM software do not entirely match the units in the database. For example, most of OSB sheathing is provided in $4 \mathrm{ft} \times 8 \mathrm{ft}$ piece, so each piece has an area of $32 \mathrm{sq} \mathrm{ft}$. In this regard, the sheathing quantity from the BIM 3D model needs to be divided by 32 to convert the unit from square feet to piece. The situations mentioned above are common in reality, so to procure an applicable quantity, an auxiliary property of material is added to the database called "Raw Quantity" $\left(Q_{R}\right)$. This property is designated to hold the extracted quantities without any extra processes temporarily, and the converted quantity is transferred to the property of "Quantity" $(Q)$ by adding equations in the database (Figure 5).

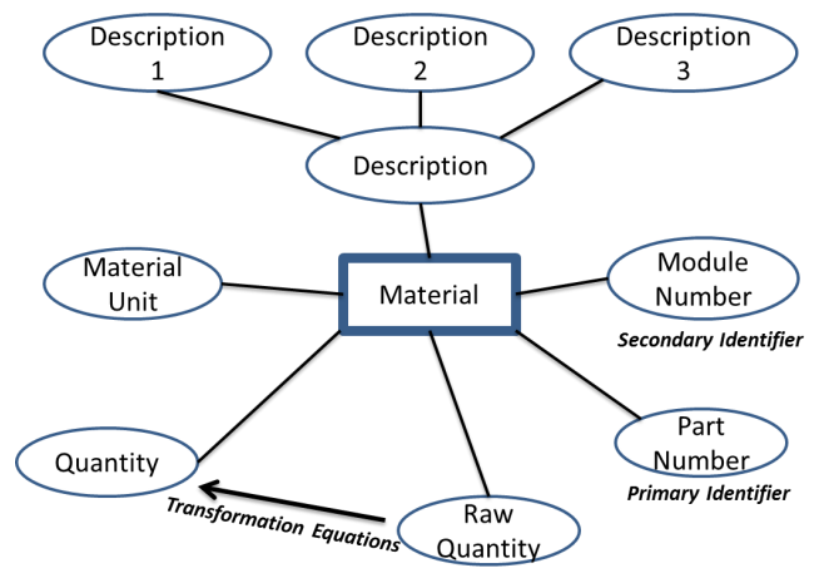

Figure 5. Database property structure

$$
\begin{gathered}
Q=f\left(Q_{R}\right) \\
Q=Q_{R} * f_{u} *\left(1+f_{w}\right) *\left(1+f_{c}\right)
\end{gathered}
$$

Where $Q$ is the final quantity; $Q_{R}$ is the raw quantity without processes; $f_{w}$ is the material waste factor; $f_{c}$ denotes construction factor; and $f_{u}$ represents unit conversion factor.

\section{Quantity Extraction}

After the BIM 3D model and database template are pre-loaded with the required information, the quantities are ready to be extracted according to different categories $(\mathrm{C} 1, \mathrm{C} 2$, and $\mathrm{C} 3)$. All the materials in the BIM 3D model are collected and the materials with the identical "Part Number" 
and "Module Number" are accumulated to generate the quantity, which is extracted to database, and assigned to the property of "Raw Quantity". In Category 1, the quantity is the sum of the material counts. In terms of Category 2 , the material lengths $\left(l_{\left(p_{i} \& m_{J}\right)}\right)$ are extracted and accumulated in order to obtain the quantity (see equation 8). For Category 3, the material areas $\left(a_{\left(p_{i} \& m_{J}\right)}\right)$ are extracted and accumulated in order to obtain the quantity. As the quantities are assigned to the property of "Raw Quantity", the front loaded formulas simultaneously calculate and transfer the processed values to "Quantity", which is regarded as the final output of quantity takeoff.

$$
\begin{aligned}
& L_{\left(p_{i} \& m_{J}\right)}=\sum_{\substack{0 \leq i<m \\
0 \leq j<n}} l_{\left(p_{i} \& m_{J}\right)} \\
& A_{\left(p_{i} \& m_{J}\right)}=\sum_{\substack{0 \leq i<m \\
0 \leq j<n}} a_{\left(p_{i} \& m_{J}\right)}
\end{aligned}
$$

Where $p_{i}$ is the part number of a material; $m_{j}$ is the module number of a material belongs to; $m$ is the quantity of different types of materials in the BIM 3D model; and $n$ denotes the quantity of modules in the BIM 3D model.

\section{CASE STUDY:}

To illustrate the effectiveness of the proposed methodology, an actual case study is presented. The case study object is a recreation facility with $72 \mathrm{ft}$ in length and $24 \mathrm{ft}$ in width, and it combined by two $72 \mathrm{ft}$ by $12 \mathrm{ft}$ modules, and a common wall between two modules is opened up to obtain a large open space. Two pool tables, two Texas Holdem Poker tables and two table tennis tables are placed in the modules, and a stair is installed outside the module in order to provide an access (Figure 6). The case study demonstrates how the method is performed to extract the material quantities from a 3D model in Revit, and display the quantity takeoff in an Excel spreadsheet.

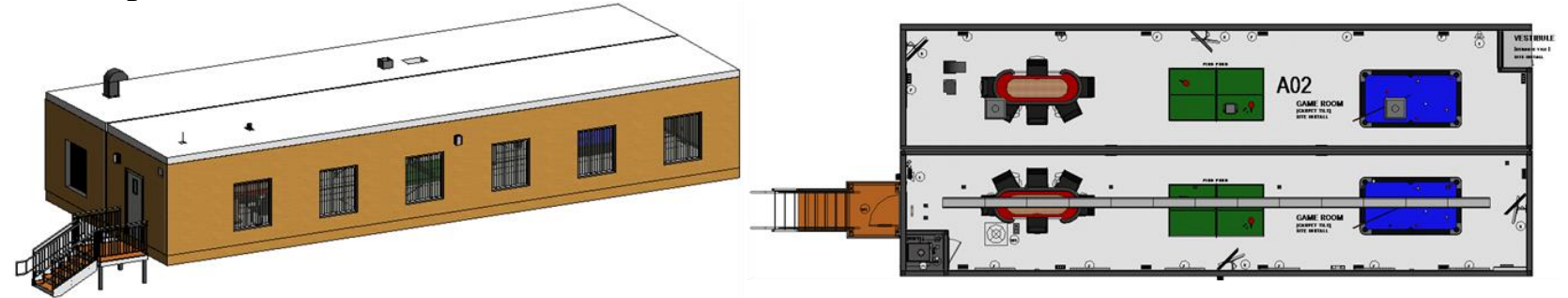

Figure 6: $3 \mathrm{D}$ view and top view of the case study model

\section{Preload Revit Model}

The BIM model of this project as shown in Figure $6 \mathrm{c}$ is developed in Autodesk Revit, and it consists of two modules. Each is assigned a distinct "Module Number". The upper module is assigned as "Unit 02", and the lower one is assigned as "Unit 01 ". The materials belong to each module have the identical "Module Number" as the module. Besides the modules, there is a stair which is shared by two modules, and the stair does not belong to any particular module, despite that it connects with "Unit 01" module. Module number of the stair is thus assigned as "Site Install". In this scenario, each material in the model has a 6-digit "Part Number" (called JDE\# in Civeo), and "Module Number" is named "Unit Number", and the combination of "JDE\#" and "Unit Number" is the bridge to integrate Revit model with Excel template and the key parameter to mirror the quantities onto the Excel. Figure 7 presents the preloaded information in the given BIM model. 


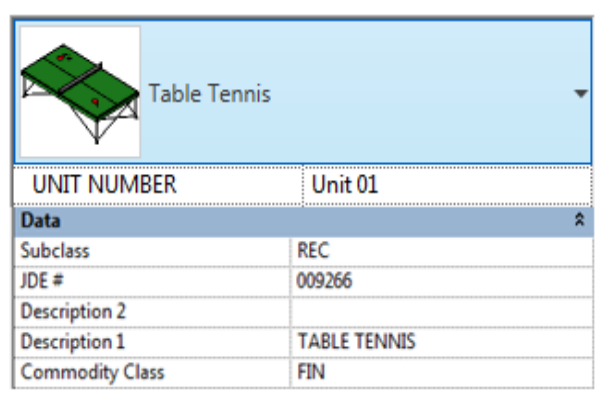

Figure 7. Preloaded information in the Revit model

\section{Standardization of Excel Template}

The properties of material are transformed to be the headers in the Excel template. This standardized Excel template contains the following columns, "Part_Number", "Description1", "Description2", "Unit", and "Unit_Number", which are filled up in advance. Another two columns "Raw Quantity" and "Quantity" are blank to hold quantity extractions. Since three different units ("Unit 01", "Unit 02" and "Site Install") exist in the model, the content in the Excel template are duplicated twice, indicating that there are three identical groups of materials exist in the template, and "Unit 01", "Unit 02", and "Site Install" are assigned to each group under "Unit_Number" column (Table 2).

Table 1. Sample in the Excel template

\begin{tabular}{|c|c|c|c|c|c|c|c|}
\hline Item_Number & Quantity & Unit & Description1 & Description2 & Unit_Nunmber & Family_Name & Raw_Quantity \\
\hline 009266 & & EA & TABLE TENNIS & & Unit 01 & & \\
\hline 009266 & & EA & TABLE TENNIS & & Unit 02 & & \\
\hline 009266 & EA & TABLE TENNIS & & Site Install & & \\
\hline
\end{tabular}

\section{Quantity Extraction}

In the Autodesk Revit, all the material information are collected, which includes the part numbers, unit numbers, and quantities. As long as more than one item has the same part number and unit number, they are grouped with a quantity of the sum. The prototype automatically searches the entire Excel template to sum up all the cells matching with identical part number and unit number under the column of "Raw_Quantity". Due to the fact that formulas are preloaded into in the Excel, the number under "Raw_Quantity" is modified by this formula and transported to the cell under column "Quantity" in the same row simultaneously (Table 3).

Table 3. Extracted quantities in the Excel template

\begin{tabular}{|c|c|c|}
\hline JDE_Number & Quantity & Un \\
\hline 011830 & 6 & EA \\
\hline 022319 & 2 & EA \\
\hline 004971 & 117.5966 & EA \\
\hline 044566 & 0.594044 & EA \\
\hline
\end{tabular}

\begin{tabular}{|c|c|c}
\hline Unit & Description1 & \\
\hline EA & RECP 15A 125V DUPLEX DECORA & \\
\hline EA & SWITCH 15A 120V DECORA 3P & WH \\
\hline EA & LUM SPF 2X6X10FT & \\
EA & TYVEK HOME WRAP & \\
\hline
\end{tabular}

\begin{tabular}{|c|c|c|}
\hline Description2 & Unit_Number & Raw Quantity \\
\hline LEVITON 5325-W & Unit 01 & 6 \\
\hline HITE LEVITON 5603-P2W & Unit 01 & 2 \\
\hline \#2\&BTR & Unit 01 & 1069.06 \\
\hline 10FT X 150FT & Unit 01 & 645.7 \\
\hline
\end{tabular}

The first two items (receptacle and switch) in the sample output belong to Category 1, which is counted by each. Hence, the directly extracted quantity under the column "Raw Quantity" can be transferred to the column "Quantity" without any processes. The third item (lumber 2x6x10 ft) and last item (Home Wrap) belong to Category 2 and Category 3 respectively, so the formula is requisite to transform the "Raw Quantity". For Category 2, it is uncontested to assume the extracted lumber length accords with the final lumber length in real construction; as a result, the construction factor $f_{c}$ is 0 . However the waste is inevitable due to the cutting process and the company always issues extra $10 \%$ to $15 \%$ materials for conservative estimate, which leads to the 
waste factor $f_{w}$ of $10 \%$ for lumbers and other Category 2 materials. Moreover, since the unit piece of regular lumber is dimensioned by $10 \mathrm{ft}$, the unit conversion factor $f_{u}$ is set to be $1 / 10$ to convert the extracted unit "ft" to inventory unit "Each". The final value after calculation is 117.60, with the raw quantity of 1069.06 shown in Table 3. As described in the previous section, the material waste from the home wrap placement on the walls with openings is understandable and unavoidable, and the waste causes the material quantity deviation between 3D model and reality, therefore, the construction factor $f_{c}$ is valuated to be $20 \%$ to eliminate the measurement discrepancy. The stocked home wrap is in the dimension of $10 \mathrm{ft}$ x $150 \mathrm{ft}(1500 \mathrm{sq} \mathrm{ft})$, so the unit conversion factor $f_{u}$ is set to be $1 / 1500$, and the waste factor $f_{w}$ is assumed to be $15 \%$. Accordingly, the final formula is established as below.

$$
Q_{\text {wrap }}=\text { Quantity }_{\text {raw }} *(1 / 1500) *(1+15 \%) *(1+20 \%)=0.59
$$

\section{CONCLUSION}

The study presents a methodology for automatic quantity takeoff. In the methodology, a unique classification system (e.g. part number in this study) is pre-loaded into BIM model and Excel template in order to facilitate the automatic quantity take-off. All quantities are extracted and quantified in according with unique classification system in BIM model and exported into the excel database. All information exported into excel is organized in the format of unique classification system, which provides end-users an opportunity of obtaining derived quantities. A Revit add-on is developed to implement the proposed methodology through Revit Application Programming Interface. Additionally, a user-friendly graphic interface has been developed in order to make the prototype system to be easily adopted into the current industry. A case example of a modularized house is adopted to demonstrate the proposed methodology and the prototyped system. The result reveals that a considerable amount of time saving and accuracy increasing of project estimation are achieved by means of the automated quantity takeoff. The successful implementation demonstrated the practical usefulness of the prototyped system.

\section{ACKNOWLEDGEMENTS}

We gratefully acknowledge the financial support of the Natural Sciences and Engineering Research Council of Canada (NSERC), as well as the assistance from Civeo, Edmonton, Canada.

\section{REFERENCES}

Autodesk Quantity Takeoff. (2015). Retrieved February 9, 2015, from http://usa.autodesk.com/adsk/servlet/pc/index?id=10354648\&siteID=123112

Considerations for assessing the benefits of standardisation and pre-assembly in construction. (2002). Retrieved March 10, 2015, from https://dspace.lboro.ac.uk/dspacejspui/bitstream/2134/9020/1/2002 Pasquire Gibb J-FMPC SP paper.pdf

Eastman, C., Teicholz, P., Sacks, R., and Liston, K.(2008). BIM handbook: A guide to building information moedling for owners, managers, designers, engineers and contractors, Wiley, New York.

Safety Hazards to Workers in Modular Home Construction . (2003). Retrieved March 10, 2015, from http://www.cpwr.com/sites/default/files/publications/becker_modhomes.pdf

Tyvek Housewrap Breather Membrane for wall condensation protection. (2014). Retrieved February 2, 2015, from http://www.just-insulation.com/002-brands/tyvek-housewrap-breathermembrane-for-walls.html 